\section{ESSENTIALS OF ORAL HYGIENE}

The Dental Channel's Caring For Your Mouth DVD is a dental health education title produced in association with the British Dental Health Foundation. Centred around ten concise videos, it is designed to help dentists, hygienists and health educators inform their patients about the essentials of oral hygiene and related subjects quickly, easily and with minimum fuss.

The on-demand webinar catalogue includes Oral Health Education (1.5 hrs vCPD) and Oral Health Education - Dietary Analysis (1.5 hrs vCPD).

Access to these and all other ondemand presentations for the duration of 2010 is available now for $£ 100.00$ +VAT - currently providing over 60 hours of verifiable CPD.

On-demand courses are accessed online at www.dental-channel.co.uk and can be purchased individually or as part of a package.

Verifiable CPD is obtained from all of the Dental Channel's courses, providing an easy and relaxed approach to keeping up to date with new knowledge and best practice in all things dental.

Reader response number 60

\section{EFFECTIVELY TACKLING BACTERIA}

The Curasept range from Curaprox features the anti-microbial qualities of chlorhexidine, without the conventional side effects of altered taste perception as well as staining teeth.

The range is specially formulated to leave teeth free from stains. Other benefits include added fluoride, making Curasept ideal for ongoing use as part of a daily oral hygiene routine. It includes a mouthwash in two strengths: 0.05\% chlorhexidine for daily use, or $0.2 \%$ chlorhexidine, suitable for daily use for up to two-week periods. Both are alcohol-free so there is no concern about the potential link with mouth cancer and alcohol. There is also Curasept tooth-

\section{MAKING ORDERING QUICKER}

Henry Schein Minerva's eZscan is a time-saving innovation designed to enhance the sale of oral hygiene products, enabling any practice to easily generate additional revenue streams and giving patients the full benefit of professional recommendation.

The barcode reading device is capable of measuring and monitoring stock levels ensuring that merchandising units are paste, containing $0.05 \%$ chlorhexidine and $0.05 \%$ fluoride, ideally used in conjunction with the mouthwash for optimum benefit. Curasept Gel is for topical application directly to the gums for an effective way to tackle the bacteria in the mouth.

\section{Reader response number 61}

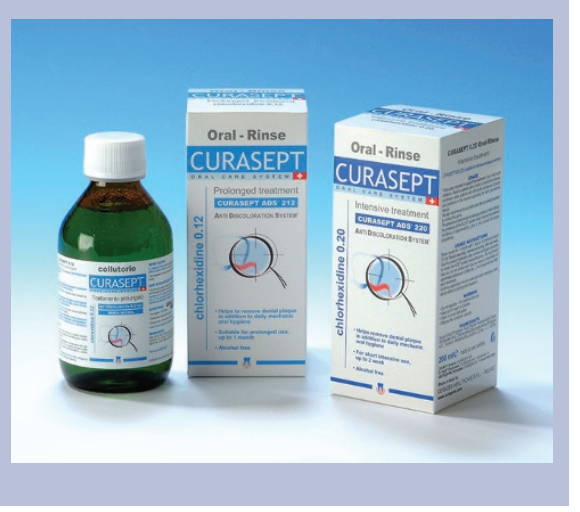

\title{
DETECTS CALCULUS WITHOUT HARMING HEALTHY TEETH
}

Sident Dental Systems' PerioScan is the new innovative ultrasonic scaler from Sirona.

It not only removes, but also detects calculus without harming healthy teeth. The Base unit and handpiece have coloured lights which clearly indicate its presence. Green signifies healthy root surface and blue calculus. This makes treatment more efficient and eliminates the potential for insufficient or excessive treatment.

Operators can complete all stages of treatment with one instrument, without any changes. The base unit's screen shows all the settings and a picture of a tooth which changes colour when calculus is present. It also has three educational images which can be used to explain treatment to patients.

Clinicians can select either water, saline or chlorhexidine as the irrigant - and alter the power setting for the scaler tip before or during treatment. Its handpiece is ergonomically designed to fit the operator's hand and has a light ring which changes colour when calculus is present. This provides a visual indication of calculus to reinforce tactile perception. An optional acoustic setting also provides an audible indicator of calculus. The footswitch has three roles: it starts treatment, can initiate irrigation without ultrasonics and boosts performance by $20 \%$.

Reader response number 63 kept well-stocked with the items you sell most. eZscan allows you to simply scan the barcodes of the products you would like to order, either from previous stock or by using the Henry Schein Minerva catalogue that is fully bar-coded for added convenience. By connecting the scanner directly to your PC you can upload your completed list, making ordering quick, easy and preventing errors.

Reader response number 62 